\title{
In situ Correlative Helium Ion Microscopy and Secondary Ion Mass Spectrometry for High-Resolution Nano-Analytics in Life Sciences
}

\author{
Jelena Lovric ${ }^{1 *}$, Jean-Nicolas Audinot ${ }^{1}$ and Tom Wirtz ${ }^{1}$ \\ 1. Advanced Instrumentation for Ion Nano-Analytics, Department of Materials Research and \\ Technology, Luxembourg Institute of Science and Technology (LIST), Belvaux, Luxembourg. \\ * Corresponding author: jelena.lovric@list.lu
}

In numerous research areas development of innovative characterization techniques at nano-scale is of great importance to advance the frontiers of science. The Helium Ion Microscope (HIM) has emerged as an instrument of choice for high-resolution imaging. Secondary electron (SE) based imaging can be performed on the HIM with resolutions down to 0.5 and $2 \mathrm{~nm}$ when scanning the sample with $\mathrm{He}^{+}$and $\mathrm{Ne}^{+}$beams, respectively. The combination between probing the sample with light ions and detecting emitted electrons confers unique capabilities to the HIM, including high contrast imaging and high depth of field [1].

Although HIM offers an excellent spatial resolution, until recently it could not provide chemical analyses. In contrast to Scanning Electron Microscopy (SEM), chemical or elemental information cannot be obtained on the HIM by using X-ray spectroscopy, as the velocity of $30 \mathrm{keV} \mathrm{He}^{+}$or $\mathrm{Ne}^{+}$ions is too small to generate a useful $\mathrm{x}$-ray signal from the sample. However, various alternative analytical methods along with appropriate instrumentation have been developed in order to obtain chemical information on HIM. In particular, it is possible to perform Secondary Ion Mass Spectrometry (SIMS) on the HIM as the impinging $\mathrm{He}^{+}$or $\mathrm{Ne}^{+}$ions (primary ion beam) typically used for SE imaging are sputtering the sample surface and are thereby leading to the emission of secondary ions that can be analysed in a mass spectrometer [2-5]. SIMS is a powerful technique for chemical analyses of surfaces owing to its excellent sensitivity, high dynamic range (a same signal can be followed over several orders of magnitude), high spatial and mass resolution. One of the main advantages of the HIM-SIMS technique with its high-spatial resolution capability (sub-nm secondary electron imaging combined with sub-20 nm SIMS imaging) is the possibility to achieve in-situ correlative imaging. This approach allows SE images of exactly the same zone analyzed with SIMS to be acquired easily and rapidly, followed by an image fusion between the SE and SIMS data sets [6] (Fig. 1). The integrated approach allows fast and multiple interlacing between the different imaging and analysis modes reducing significantly the analysis time.

In this work, we focus on the application of the HIM-SIMS technology in different life science research fields. As a first step, we investigated the methodology to analyse a typical biological sample consisting of a chemically fixed and resin embedded bacteria exposed to nanoparticles. The standard HIM imaging by detecting secondary electrons of thin flat sections showed difficulties to reveal sample features. However, when using the SIMS system to detect all negatively charged particles (electrons and negative ions) emitted from the sample, more structural details were obtained. Secondary ion maps of endogenous $\mathrm{CN}^{-}$and $\mathrm{Cl}^{-}$ions and ion signal originating from NPs $\left(\mathrm{Ti}^{+}\right)$were acquired allowing the interaction between bacterial cells and NPs to be assessed (Fig. 1). Moreover, we optimised the sample preparation and imaging methodology in order to obtain high-resolution secondary electron images and correlate these with high-spatial resolution SIMS imaging of specific cell features highlighted using labelling with halogens. 
Recently, our research focus has been directed towards the development of the npSCOPE instrument, a new integrated instrument optimised for physico-chemical characterisation of primarily nanoparticles in biological samples. Beside the possibility to perform correlative SE and SIMS imaging, this instrument will allow obtaining structural data with in-situ Scanning Transmission Ion Microscopy (STIM). Finally and of utmost importance, the instrument will operate in a cryo-mode allowing analysis of biological samples in frozen-hydrated state and thus avoiding artefacts from a classical sample preparation used for UHV imaging at room temperature [7].

We show that the distinctive capability of HIM-SIMS in terms of correlative microscopy combining both high-spatial resolution structural information and high sensitivity chemical information allows research in various areas of life sciences such as nanotoxicology, virology and lipid research, subcellular and sub-organelle analyses, and potentially many others [8].

References:

[1] BW Ward, JA Notte and NP Economou, Journal of Vacuum Science \& Technology B 24 (2006), p. 2871.

[2] T Wirtz, D Dowsett and P Philipp in "SIMS on the Helium Ion Microscope: A Powerful Tool for High-Resolution High-Sensitivity Nano-Analytics". In: Hlawacek G., Gölzhäuser A. (eds) Helium Ion Microscopy (Springer, Cham) p. 297.

[3] T Wirtz et al., Nanotechnology 26 (2015), p. 434001.

[4] D Dowsett and T Wirtz, Analytical Chemistry 89 (2017), p. 8957.

[5] T Wirtz et al., Ann Rev of Analytical Chemistry 12 (2019).

[6] F Vollnhals et al., Analytical Chemistry 89 (2017), p. 10702.

[7] npSCOPE, https://www.npscope.eu/

[8] This project has received funding from the European Union's Horizon 2020 Research and Innovation Programme under grant agreement No. 720964. The authors kindly acknowledge M. Mercier-Bonin, Toxalim, France, P. Chlanda, University Hospital Heidelberg, Germany and S. Jiang and X. RoviraClave, Stanford University, USA for providing biological samples.
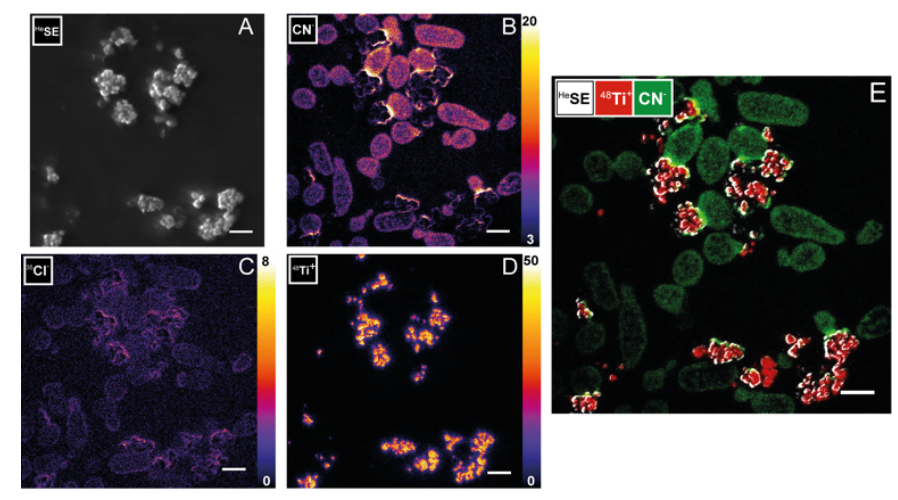

Figure 1. HIM-SIMS analysis of E. coli bacteria exposed to $\mathrm{TiO}_{2}$ nanoparticles: A. SE image; B-D. SIMS images of $\mathrm{CN}^{-}, \mathrm{Cl}^{-}$and $\mathrm{Ti}^{+}$, respectively; E. Laplace pyramid image fusion of structural (SE) and chemical (SIMS) information. SE: $20 \mathrm{keV} \mathrm{He}{ }^{+}, \mathrm{I}_{\mathrm{p}}=11.7 \mathrm{pA}, 1024 \times 1024$ pixels, $20 \mu \mathrm{s} /$ pixel; SIMS: 20 $\mathrm{keV} \mathrm{Ne}^{+}, \mathrm{I}_{\mathrm{p}}=8 \mathrm{pA}, 512 \times 512$ pixels, $3 \mathrm{~ms} /$ pixel; Scale bar: $1 \mu \mathrm{m}$. 